\title{
Genome-wide analyses of the relict gull (Larus relictus): insights and evolutionary implications
}

\author{
Chao Yang ${ }^{1,2+}$, Xuejuan $\mathrm{Li}^{1 \dagger}$, Qingxiong Wang ${ }^{2}$, Hao Yuan ${ }^{1}$, Yuan Huang ${ }^{1 *}$ (D) and Hong Xiao ${ }^{2^{*}}$
}

\begin{abstract}
Background: The relict gull (Larus relictus), was classified as vulnerable on the IUCN Red List and is a first-class national protected bird in China. Genomic resources for L. relictus are lacking, which limits the study of its evolution and its conservation.

Results: In this study, based on the Illumina and PacBio sequencing platforms, we successfully assembled the genome of $L$. relictus, one of the few known reference genomes in genus Larus. The size of the final assembled genome was $1.21 \mathrm{~Gb}$, with a contig N50 of $8.11 \mathrm{Mb}$. A total of 18,454 genes were predicted from the assembly results, with 16,967 (91.94\%) of these genes annotated. The genome contained $92.52 \mathrm{Mb}$ of repeat sequence, accounting for $7.63 \%$ of the assembly. A phylogenetic tree was constructed using 4902 single-copy orthologous genes, which showed L. relictus had closest relative of L. smithsonianus, with divergence time of 14.7 Mya estimated between of them. PSMC analyses indicated that L. relictus had been undergoing a long-term population decline during 0.01-0.1 Mya with a small effective population size fom 8800 to 2200 individuals.
\end{abstract}

Conclusions: This genome will be a valuable genomic resource for a range of genomic and conservation studies of $L$. relictus and will help to establish a foundation for further studies investigating whether the breeding population is a complex population. As the species is threatened by habitat loss and fragmentation, actions to protect $L$. relictus are suggested to alleviate the fragmentation of breeding populations.

Keywords: Whole-genome, PacBio sequencing, Larus relictus, Habitat loss, Population fragmentation

\section{Background}

The relict gull (Larus relictus) (Charadriiformes, Laridae, Larus), a middle-sized gull with a black-coloured head, had been known for nearly 50 years before it was regarded as a unique species [1]. It is classified as vulnerable (VU) on the IUCN Red List and is a first-class national protected bird in China. Its population size has been estimated at 10,000-19,999 (BirdLife International, $2020)$, and the vast majority of L. relictus $(90 \%)$ reside in Hongjian Nur with very low genetic diversity [2]. Their

\footnotetext{
* Correspondence: yuanh@snnu.edu.cn; xh4500@163.com

${ }^{\dagger}$ Chao Yang and Xuejuan Li contributed equally to this work.

${ }^{1}$ College of Life Sciences, Shaanxi Normal University, Xi'an 710062, China

${ }^{2}$ Shaanxi Institute of Zoology, Xi'an 710032, China
}

main wintering place is situated on the west coast of the Bohai Sea [3]. A small number of winter migratory individuals have been sighted in Hong Kong [4]. Therefore, the main threats to $L$. relictus are lake shrinkage on breeding grounds and at stopover sites, as well as the loss of intertidal flats on wintering grounds [5]. A novel data-driven habitat suitability ranking approach for $L$. relictus using remote sensing and GIS indicated that three threat factors, road networks, developed buildings and vegetation, affect suitable habitat for this species most severely [6].

On the whole-genome level, DNA sequencing technology is usually used to characterize genetic variation and acquire comprehensive molecular characterizations [7].

(c) The Author(s). 2021 Open Access This article is licensed under a Creative Commons Attribution 4.0 International License, which permits use, sharing, adaptation, distribution and reproduction in any medium or format, as long as you give appropriate credit to the original author(s) and the source, provide a link to the Creative Commons licence, and indicate if changes were made. The images or other third party material in this article are included in the article's Creative Commons licence, unless indicated otherwise in a credit line to the material. If material is not included in the article's Creative Commons licence and your intended use is not permitted by statutory regulation or exceeds the permitted use, you will need to obtain permission directly from the copyright holder. To view a copy of this licence, visit http://creativecommons.org/licenses/by/4.0/. The Creative Commons Public Domain Dedication waiver (http://creativecommons.org/publicdomain/zero/1.0/) applies to the data made available in this article, unless otherwise stated in a credit line to the data. 
At present, only limited genetic information, in the form of mitochondrial markers and inferred population structure, is available for $L$. relictus [2, 8-10]. However, no genome has been published for $L$. relictus which limits our understanding about the molecular mechanisms of evolutionary and genetic processes.

High-throughput sequencing technology has notably reduced sequencing costs [11] and marked the start of a new era of genomic studies [12]. Among them, longread sequencing technologies such as Pacific Biosciences (PacBio) [13] can produce average read lengths of over 10,000 bp [12]. PacBio technology has been used to obtain high-quality genome assemblies for several avian species, such as Gallus gallus (Galliformes) [14] and Malurus cyaneus (Passeriformes) [15].

In this study, the first contig-level genome of $L$. relictus was constructed using both Illumina HiSeq and PacBio sequencing platforms. We assessed various genomic characteristics and performed comparative analyses. These genomic data will facilitate population studies of L. relictus and support the comprehensive protection of this vulnerable avian species.

\section{Results}

\section{Genome sequencing and assembly}

Approximately $106.29 \mathrm{~Gb}$ of raw sequencing data were obtained using the Illumina HiSeq platform, including three 250-bp insert libraries and two 350-bp insert libraries (Table S1). The sequencing depth was 87.85X. We used the PacBio sequencing platform with three $20-\mathrm{Kb}$ libraries to obtain long reads for assembling the genome and retained approximately $30.50 \mathrm{~Gb}$ raw data. The sequencing depth was 25.42X. After filtering out low-quality and short-length reads, the read N50 and mean read length were 12,712 bp and 8418 bp, respectively (Table S2, S3). Finally, a 1.21 Gb assembly with a contig N50 of approximately $8.11 \mathrm{Mb}$ was obtained for $L$. relictus, with a GC content of approximately $43.11 \%$. The genome consisted of 1313 contigs, with the longest contig being approximately 29.7 Mb long (Table S4).

Approximately $99.96-99.97 \%$ of the cleand Illumina reads could be mapped to the contigs, with $93.33-93.77 \%$ properly mapped reads (Table S5). The CEGMA v2.5 analysis identified 416 core eukaryotic genes (CEGs), accounting for $90.83 \%$ of all 458 CEGs, and 175 CEGs (70.56\%) could be detected with homology to the 248 highly conserved CEGs (Table S6). In addition, 4555 (92.7\%) of the 4915 highly conserved Aves orthologues from BUSCO v3.0.2 were identified in the assembly (Table S7). These results show that the assembled $L$. relictus genome sequence was complete and had a low error rate.

\section{Genome annotation}

The consensus gene set included a total of 18,454 genes were predicted by three different strategies (Methods section for details) (Table S8). The average gene length, exon length, and intron length were $20,749.08 \mathrm{bp}$, $164.24 \mathrm{bp}$, and $1996.77 \mathrm{bp}$, respectively. The final prediction results revealed 17,452 (94.57\%) supported by homology-based and RNA-seq-based methods (Fig. S1), which showed a good gene prediction efficiency compared to gene annotations of genomes in five known species of Laridae, human and G. gallus (Table S9) [16, 17]. A total of 16,967 (91.94\%) predicted genes in the $L$. relictus genome were annotated and functionally classified by the Gene Ontology (GO) [18], Kyoto Encyclopedia of Genes and Genomes (KEGG) [19], Cluster of Orthologous Groups for eukaryotic complete genomes (KOG) [20], Translated EMBL-Bank (TrEMBL) [21] and NCBI non-redundant amino acid sequences (NR) [22] databases (Table S10).

Noncoding RNAs were also identified and annotated, including 208 microRNA genes (miRNAs), 73 rRNAs and 289 tRNAs. A total of 221 pseudogenes were identified in the $L$. relictus genome.

A total of $92.52 \mathrm{Mb}$ of repeat sequence was annotated, composing $7.63 \%$ of the total genome length. We found that class I transposable elements (TEs) (RNA transposons or retrotransposons) occupied approximately $8.22 \%$ of the genome assembly. Among class I TEs, $1.12 \%$ were long terminal repeat elements (LTRs), 5.85\% were long interspersed elements (LINEs) and $0.02 \%$ were short interspersed elements (SINEs) (Table S11). The LINE percentage from 4.95 to $6.03 \%$ and SINE percentage from 0.1 to $0.15 \%$ in five known species of Laridae genomes, respectively [17]. While the content of SINEs in $L$. relictus were obviously less common than in Laridae and this novel phenomenon needs to be futher studied. The $L$. relictus genome also contained class II TEs (DNA transposons), which occupied approximately $0.28 \%$ of the genome.

\section{Gene families}

Comparison of the L . relictus genome assembly with the genomes of eleven other Charadriiformes species showed that a total of 14,453 genes of $L$. relictus could be clustered into 13,799 gene families, including 201 unique genes belonging to 62 gene families. The proportion of species-specific genes within L. relictus genome (1.1\%) was obviously larger than that of other sampled genomes (0.0-0.1\%) (Table S12). In addition, 5100 gene families were shared among all sampled species. The phylogenetic relationships based on 4902 single-copy orthologous genes indicated that all seven gulls were categorized into one branch, and L. relictus was genetically most related to another member of the order Laridae, $L$. smithsonianus in kinship (Fig. 1) with divergence time of 14.7 million years ago (Mya) (time 8-21 was supported by $95 \%$ highest posterior density (HPD) (Fig. 2). 


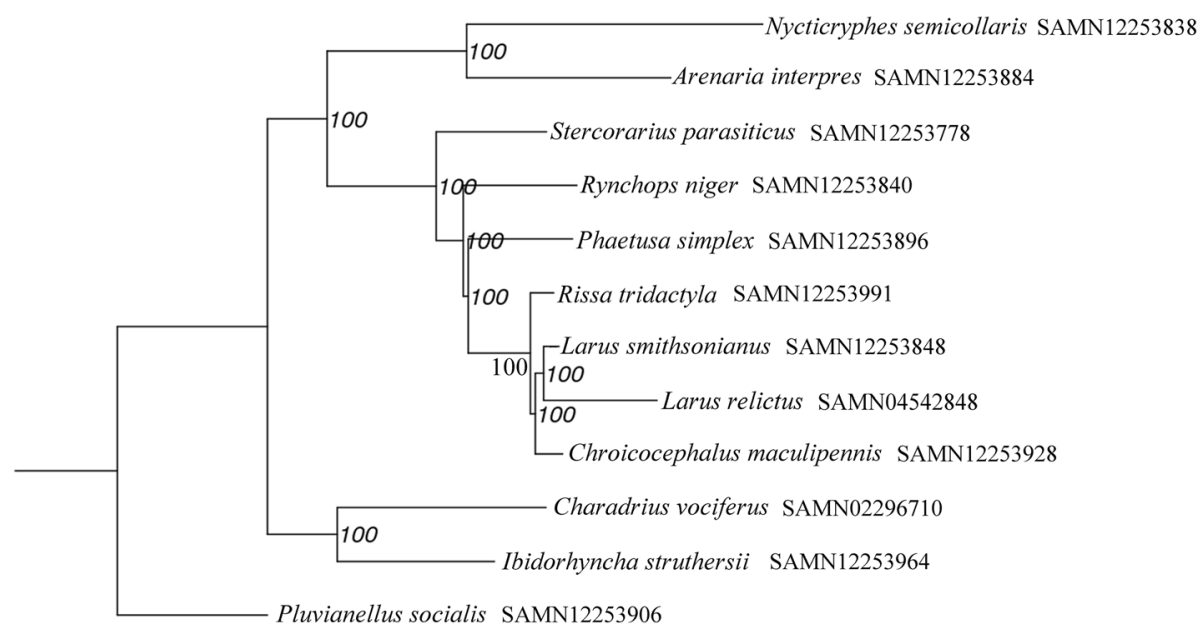

Fig. 1 Topology of Maximum likelihood (ML) tree for 12 Charadriiformes species. Tree reconstruction based on single-copy orthologues protein sequences under IQ-TREE v1.6.11. BioSample numbers are indicated following species name. Numbers on nodes are bootstrap values

\section{Positive selection genes and functional enrichment}

We found that 842 single-copy orthologous genes were under positive selection in the L. relictus genome (Table S13). The GO annotation classifies the positively selected genes (PSGs) in terms of three categories: cellular component, biological process, and molecular function. Cellular component annotations were primarily cytosol and nuclear speck. Molecular functions were mainly ATP binding and chromatin binding. Biological process annotations were mainly positive regulation of transcription from RNA polymerase II promoter and ubiquitindependent protein catabolic process. In addition, we also identified the biochemical pathways of the PSGs. The
KEGG annotation of the PSGs suggested that the pathway of RNA transport had the highest ratio, followed by spliceosome. (Fig. S2).

\section{Effective population size of $L$. relictus}

Pairwise sequentially Markovian coalescent (PSMC) analysis showed the demographic history of $L$. relictus from 100,000 years ago to 10,000 years ago. L. relictus had experienced a long period of population size decline, with the effective population size $(\mathrm{Ne})$ from approximately 8800 individuals to 2200 individuals (Fig. 3).

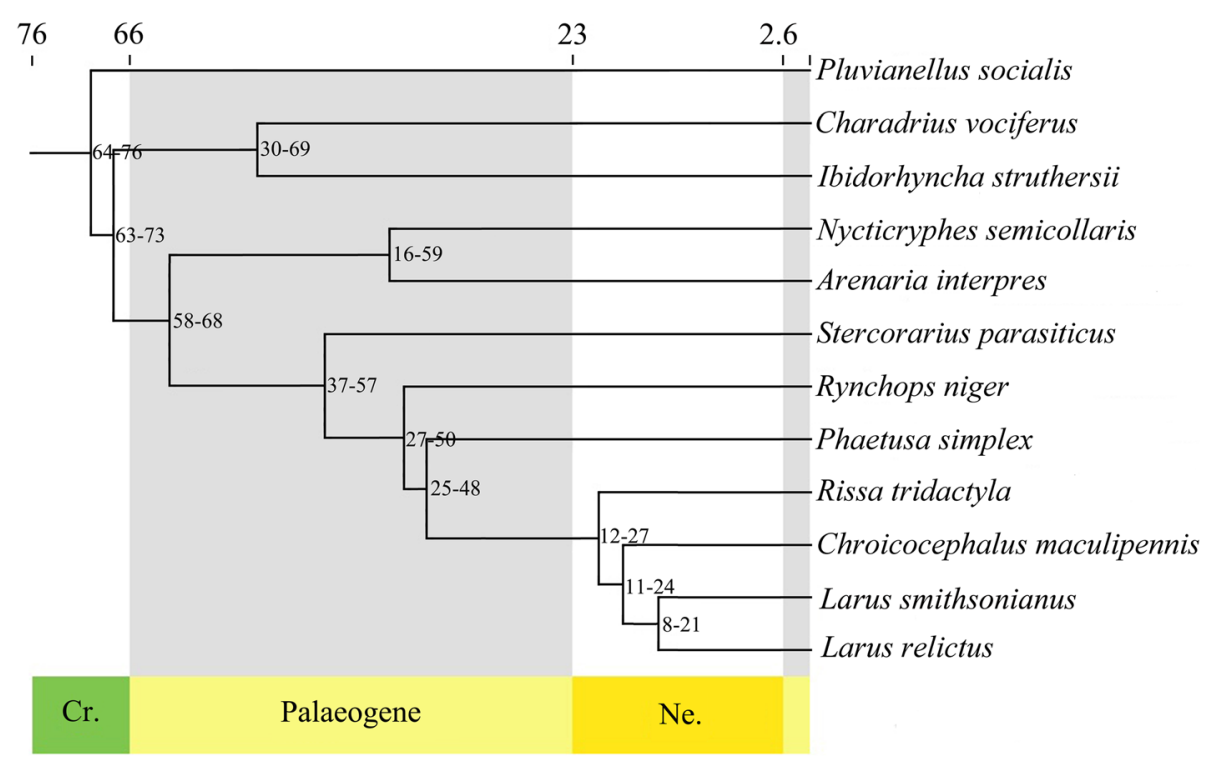

Fig. 2 Timing of inferred divergence of 12 Charadriiformes species. Numbers on the nodes represent divergence times supported by $95 \%$ HPD 


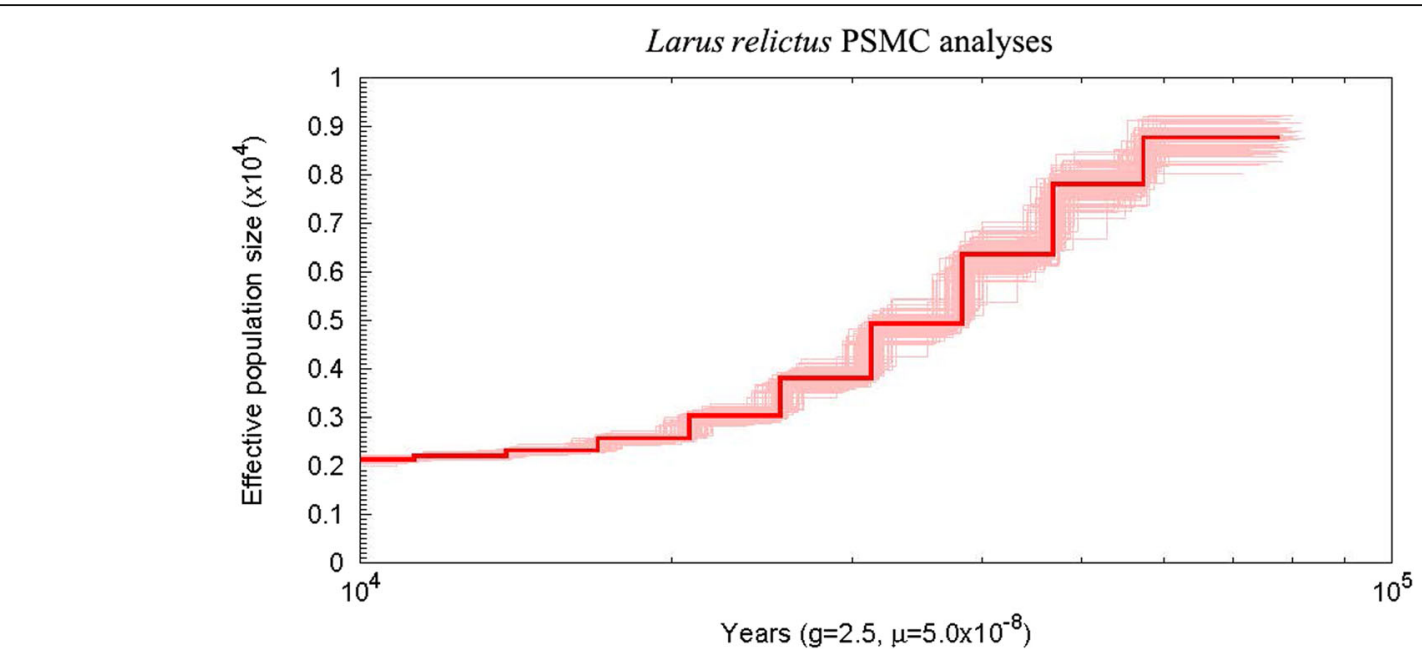

Fig. 3 The PSMC analyses result of Larus relictus. An individual re-sequencing raw data was obtained in NCBI with accession number SRR14041273. One hundred iterations were performed

\section{Discussion}

\section{Genomic characteristics}

The genome size of $L$. relictus was similar to those of five known species in Laridae, such as L. smithsonianus (1.20 $\mathrm{Gb})$. The GC content of the L. relictus genome (43.11\%) was higher than that of other known Laridae (42.2842.95\%) [17]. This proportion of repeat sequences is similar to that found in previous studies, in which almost all avian genomes contained lower levels of repeat elements than other animal genomes, with percentages of approximately 4.1-24.09\%, except for the Red-headed Barbet (Eubucco bourcierii), with approximately $29.89 \%$ of its genome, the Coppersmith Barbet (Psilopogon haemacephalus) with $31.17 \%$, and the Acacia Pied Barbet (Tricholaema leucomelas) with $31.47 \%$, respectively [16, 17]. Genomes in different vertebrate lineages can have very different contents in repeate elements: the genomes of the primates contains more repeat elements $(45-50 \%$ of the genome) than the genomes of mouse and rat (39-40\%) and $\operatorname{dog}(34 \%)[23,24]$.

\section{Topological structure and evolution}

Phylogenetic tree supported that Stercorariidae was so antiquated that it was divided out earlier than others in undergoing different selection pressures [25]. In Larus, L. relictus should be belonged to the Black-headed species, L. smithsonianus was belonged into White-headed species, but Chroicocephalus maculipennis was categorized into Masked species, respectively [26].

The timescale results indicated that the ancestral lineages of $L$. relictus and $L$. smithsonianus diverged approximately 14.7 Mya (Fig. 1). The genus Larus was split with Rissa tridactyla at approximately 20.51 Mya, which was close to that divergence time of the genus between Larus and Rissa. Pluvianellus socialis was divided out from other species were estimated at approximately 69.81 Mya, which is in agreement with the divergence time of the Charadriiformes as a whole (79-102 Mya) [27].

\section{Population dynamics}

PSMC analyses revealed that $L$. relictus had took a long period of population size decline from 0.01-0.1 Mya, with very low effective population size $0.22 \times 10^{5}-0.88 \times$ $10^{5}$ individuals (passenger pigeon, $1.3 \times 10^{5}-2.4 \times 10^{7}$ ) [28]. Decrease in genetic diversity was reflected from this phenomenon, and consistent with previous studies $(\mathrm{Pi}$, $0.00008-0.00041$ ), then leaded the loss of many alleles in the population [2]. The average estimated expansion time of L. relictus was from 0.09 to 0.23 Mya, since the late to Middle Pleistocene (0.13-0.78 Mya) and early to Late Pleistocene (0.01-0.12 Mya) [2]. Synthetic analysis, recent range expansions following recovery from a bottleneck were determined between Middle Pleistocene and Late Pleistocene. The repeated glacial-interglacial changes during the Pleistocene period (0.01-1.9 Mya) might have influenced the expansion of $L$. relictus. Neverthelessly, we infered that the population size of $L$. relictus would be going a downward trend in the end of Late Pleistocene period and early Holocene.

\section{Conclusions}

The whole-genome sequence of $L$. relictus was assembled employing the Illumina and PacBio sequencing platforms. The size of the final assembled genome was $1.21 \mathrm{~Gb}$, with a contig N50 of $8.11 \mathrm{Mb}$ and 92.52 (7.63\%) $\mathrm{Mb}$ of repeat sequence, and 18,454 genes were predicted with 16,967 (91.94\%) of these genes annotated.

Relict gull (L. relictus) has been holding a small effective population size and it has been experiencing very 
low genetic diversity and a long period of population decline while lacking a large geographical population. In this study, the genome information of L. relictus which is one of the few known reference genomes in genus Larus, will be effectively to investigate the evolutionary and molecular mechanisms of some significant processes in this species.

\section{Methods}

\section{Sampling information}

A naturally dead $L$. relictus fledgling from Hongjian Nur $\left(39^{\circ} 04^{\prime} \mathrm{N}, 109^{\circ} 53^{\prime} \mathrm{E}\right)$, Yulin, Shaanxi Province, was collected and identified by $\mathrm{H}$. Xiao, and the specimen (voucher number YG01) was deposited in the animal specimens museum of the Shaanxi Institute of Zoology, Xi'an, Shaanxi Province, China. Our team is a wildlife protection agency under the Shaanxi Academy of Sciences (China), cooperating and working with the authority department on Hongjian Nur for nearly 20 years, mainly devoted to the protection of the relict gull. To protect $\boldsymbol{L}$. relictus, this project has been approved and received permission from the Nature Reserve Authority of Hongjian Nur.

\section{DNA and RNA extraction}

DNA was extracted from the muscle using the Cetyl Trimethyl Ammonium Bromide (CTAB) method, and total RNA was extracted from the heart, liver, spleen, lung and kidney of $\boldsymbol{L}$. relictus using TRIzol reagent (Invitrogen, Carlsbad, CA, USA) following the protocol recommended by the manufacturer. DNA and RNA concentrations were measured using NanoDrop 2000, Qubit 2.0 and Agilent 2100. Only DNA with an DNA integrity number (DIN) and RNA with RNA integrity number (RIN) score $>8.0$ and $1.8<\mathrm{OD} 260 / 280<2.2$ were used for the preparation and construction of PacBio and Illumina libraries.

\section{Library preparations (DNA and RNA) and sequencing}

Both Illumina HiSeq 4000 and PacBio RSII sequencing platforms were used. For the Illumina pipeline, five short fragment paired-end libraries (three of $270 \mathrm{bp}$ and two of $350 \mathrm{bp}$ ) were constructed using the standard Illumina protocol. The details of library construction are as follows: the genomic DNA was broken randomly using the ultrasonic method, and target fragments were filtered using magnetic beads for nucleic acid purification. The small fragment sequencing library was constructed through the steps of end repair, addition of polyA and adaptor, selection of target-size fragments and PCR.

For the long fragment libraries (three of $20 \mathrm{~Kb}$ ) in the PacBio pipeline, the details of library construction are as follows: The genomic DNA was sheared using g-TUBE, followed by DNA damage-repair and end-repair. The dumbbell-type adapters were ligated, and exonuclease digestion was performed. BluePippin was used to select segments to obtain the sequencing library.

For the RNA fragment libraries (one of $280 \mathrm{bp}$ and one of MicroRNA SE50) in the Illumina pipeline, the details of library construction are as follows: Briefly, rRNA was isolated from total RNA using Epicentre Ribo-Zero ${ }^{\mathrm{mm}}$ Kit and then fragmented randomly with Fragmentation Buffer. The first-strand cDNA was synthesized with random hexamer primers using the fragmented rRNAdepleted RNA as a template, and the second-strand cDNA was synthesized with DNA polymerase I (New England Biolabs) and RNase H (Invitrogen). After end repair, A-tail, adaptor ligation and purification with AMPure XP beads, PCR amplification was conducted.

The size and quality of all constructed libraries were evaluated using an Agilent 2100, NanoDrop 2000 and Qubit 2.0. Eligible libraries were sequenced on the Illumina HiSeq 4000 platform to generate $150 \mathrm{bp}$ pairedend reads and PacBio RSII platform to generate Raw sequence data $>30.0 \mathrm{~GB}$. The Illumina HiSeq 4000 platform was also used for sequencing RNA data.

\section{Genome assembly assessment}

Raw reads were filtered to remove adapter sequences (-e 0.1 -a AGATCGGAAGAGCACACGTCTGAACT CCAGTCAC -A AGATCGGAAGAGCGTCGT GTAGGGAAAGAGTGT -m 100 --cut 0 -O 3) and lowquality data (multi_rules, $-\mathrm{u} 0.1$-q 0.5 -w 10 -Q 33 ; $\mathrm{Q} 20 / 30,-\mathrm{q} 0.95 / 0.85-\mathrm{w} 30-\mathrm{Q} 33)$, with clean reads assembled using Trinity v2.4.0 [29]. After filtering out lowquality and less than $500 \mathrm{bp}$ in length PacBio reads, LoRDEC v0.7 [30] software was used for error correction of PacBio data employing HiSeq data. The HiSeq data were preliminarily assembled by Platanus v1.2.4 [31] software. Using dbg2olc v4 [32] software, mixed assembly was carried out by using the data after error correction and the preliminary assembly results of HiSeq data. Pilon v1.22 [33] software was used to correct the assembly results using HiSeq data. To assess the completeness of the $L$. relictus genome assembly, we used two methods, with the first remapping the Illumina paired-end reads to the assembled genome and the second employing CEGMA v.2.5 [34] and BUSCO v3.0.2 databases.

\section{Genome annotation}

Methods of ab initio-based, homologue-based and RNAseq-based were used to predict gene structures, namely. EVM v1.1.1 [35] software was used to integrate the predicted genes and generate a consensus gene set. Then, GENSCAN v1.0 [36], Augustus v2.4 [37], GlimmerHMM v3.0.4 [38], GeneID v1.4 [39] and SNAP v4.0 [40] were first used to perform the ab initio prediction. For homologue prediction, GeMoMa v1.3.1 [41] was used, 
primarily employing five species as references, i.e., G. gallus, Meleagris gallopavo, Taeniopygia guttata, Ficedula albicollis and Parus major. Third, wholetranscriptomic data from the liver and an equal mix of five tissue RNA samples were used to assist genome annotations. HISAT v2.0.4 and StringTie v1.2.3 [42] were used for assembly based on RNA-seq reference data, and TransDecoder v5.0.1 [43] and GeneMarkS-T v5.1 [44] were applied to predict genes. PASA v2.0.2 [45] was used to predict unigene sequences assembled based on the whole transcriptome data without references. Finally, EVMv1.1.1 [35] was used to integrate the prediction results obtained by the above three methods, and PASA v2.0.2 [45] was used to predict alternative splice variants.

Software including LTR-FINDERv1.05 [46], MITEHunter v2011-11 [47], RepeatScout v1.05 [48] and PILE R-DF v2.4 [49] was used for prediction of repetitive sequences in the L. relictus genome. A combination of structure-based and de novo strategies was used to construct repeat databases and then merged with Repbase [50] to form a final database. RepeatMasker v4.0.6 [51] was used to identify repeat sequences with this final repeat database.

Using the Rfam [52] and miRbase [53] databases as references, rRNA and microRNA were identified by Infernal v1.1 [54]. The tRNA was predicted using tRNAscan-SE v1.3.1 [55]. GenBlastA v1.0.4 [56] was used to search homologous gene sequences on the genome whose gene loci had been shielded. Pseudogenes were then identified via GeneWise v2.4.1 [57] with premature stop codons and frame shifts.

To assign gene functions in the L. relictus genome, we aligned the genes to five functional databases using BLASTv2.2.3 [58] (E-value =1e-5). The databases included GO, KEGG, KOG, TrEMBL and NR.

\section{Phylogenetic analyses}

We used the whole-genome sequence of L. relictus and 11 published whole-genome sequences of Charadriiformes species (Arenaria interpres, Charadrius vociferous, Chroicocephalus maculipennis, Ibidorhyncha struthersii, L. smithsonianus, Nycticryphes semicollaris, Phaetusa simplex, P. socialis, R. tridactyla, Rynchops niger and Stercorarius parasiticus). Orthofinder v2.4 (diamond, $\mathrm{e}=0.001$ ) was used to cluster gene families [59]. To assign gene functions of species-specific orthogroups, we aligned the genes to GO and KEGG functional databases using clusterProfile v3.14.0 [60].

A total of 4902 single-copy orthologues were identified, with protein sequences used for constructing phylogenetic trees. The protein sequences were aligned using MAFFT v7.205 (--localpair --maxiterate 1000) [61], with PAL2NAL v14 transferred protein alignment results into codon sequences [62]. Gblocks v0.91b $(-b 5=h)$ [63] was used to remove the regions with poor alignments, and then concatenated into a combined dataset (super gene). ModelFinder was used to obtain the best model of GTR + F + I + G4 [64]. phylogenetic tree was constructed using the maximum likelihood (ML) algorithm with the JTT amino acid substitution model implemented in IQTREE v1.6.11 (bootstrap 1000) [65]. P. socialis was selected as outgroup.

Divergence times and ages of fossil records were derived from TimeTree (https://www.timetree.org/) and applied as the time control, i.e., 63.3-75.4 Mya of $P$. socialis-S. parasiticus, 59-80 Mya of L. smithsonianus- $N$. semicollaris, and 3.3-25.7 Mya of L. smithsonianus- $R$. tridactyla. Based on the results of phylogenetic tree, divergence time was estimated using the MCMCTree program in PAML v4.9i with model JC69 and correlated molecular clock. The consistency of the two repeated calculations was 1 , and iteration parameters of a Markov chain: -burnin 5,000,000 -sampfreq 30 -nsample 5,000, 000 [66]. MCMCTreeR v1.1 was used for graphical presentation.

In addition, the CodeML program in PAML v4.9i [66] included single-copy genes (F3x4 model of codon frequencies) was used to detect positively selected genes in the clade containing L. relictus, L. smithsonianus, $C$. maculipennis, $R$. tridactyla and P. simplex. Among them, the branch-site model was used, and likelihood ratio tests (LRTs) were calculated $(P<0.01)$ between Model A (foreground clade $\omega>1$ ) and null Model (any sites forbidden $\omega>1$ ). Posterior probability was calculated in Bayes empirical Bayes method (BEB).

\section{PSMC analyses}

Consensus sequences of an individual re-sequencing (average depth: 29X; coverage ratio 10X: 92.44\%) were called (SNP calling) using SAMtools v1.12, then converted into the fastq format using BCFtools v1.10 and Vcfutils (varFilter -D100 > var.flt.vcf). Bases of low sequencing depth (less than a third of the average depth) or high depth (twice the average depth) were masked. Sequences were split into short segments of $50 \mathrm{~kb}$ to estimate the demographic history with the Hidden Markov Model (HMM) model in PSMC v4.0.22 following parameters of -N25 -t15 -r 5 -b -p $(4+25 \times 2+4+6)$ [67]. The generation time $(\mathrm{g}=2.5)$ and mutation rates per year $\left(\mathrm{u}=5 \times 10^{-8}\right)$ were used. One hundred bootstraps were performed.

\section{Abbreviations}

PacBio: Pacific Biosciences; CEG: Core Eukaryotic Gene; GO: Gene Ontology; KEGG: Kyoto Encyclopedia of Genes and Genomes; KOG: Cluster of

Orthologous Groups for eukaryotic complete genomes; TrEMBL: Translated EMBL-Bank; NR: NCBI non-redundant amino acid sequences;

miRNAs: microRNA genes; TEs: Transposable Elements; LINEs: Long Interspersed Elements; LTRs: Long Terminal Repeats; SINEs: Short Interspersed Elements; Mya: Million years ago; HPD: Highest Posterior Density; 
PSGs: Positively Selected Genes; PSMC: Pairwise Sequentially Markovian Coalescent; CTAB: Cetyl Trimethyl Ammonium Bromide; DIN: DNA Integrity Number; RIN: RNA Integrity Number; LRTs: Likelihood Ratio Tests; BEB: Bayes Empirical Bayes method

\section{Supplementary Information}

The online version contains supplementary material available at https://doi. org/10.1186/s12864-021-07616-z.

Additional file 1: Figure $\mathbf{S 1}$. The results of gene prediction using three methods.

Additional file 2: Figure S2. The GO and KEGG annotation of PSGs. Only 10 items with the smallest $p$-value are shown.

Additional file 3: Table S1. Sequencing data by using Illumina platform. Table S2 Raw data filtering by using PacBio platform. Table S3 Statistics of subresds length distribution by using PacBio platform. Table S4. Statistics of genome assembly. Table S5. The mapped results using Illumina clean reads. Table S6. Statistics of genome assembly by using CEGMA v2.5. Table S7. Genome completeness assessment employing BUSCO v3.0.2. Table S8. Statistics of gene prediction. Table S9. Statistics of gene information from 10 species. Table $\mathbf{5 1 0}$. Statistic information of gene function annotation. Table S11. Repeat elements in the genome. Table S12. Classification and statistics of gene families.

Additional file 4: Table S13. Statistics of Larus relictus positively selected genes.

\section{Acknowledgements}

We thank Tuokao Han for assisting us in collecting specimens and Liliang Lin for assisting us in plotting data.

\section{Authors' contributions}

CY collected the sample, carried out all experiments, and wrote this paper. XJL analyzed sequencing data, and embellished the article. QXW collected the sample, and assisted in the programming. HY analyzed sequencing data. $\mathrm{HX}$ conceived this idea and identified the sample. YH initiated this project and refined it, revised and approved the final manuscript.

\section{Funding}

This work was supported by the National Natural Science Foundation of China (Grant No. 31601846 and 31801993), Natural Science Foundation of Shaanxi Province, China (Grant No. 2017JQ3014 and 2020JM-270), Projects for Department of Science and Technology of Shaanxi Province, China (Grant No. 2018ZDXM-NY-071 and 2019NY-089), Fundamental Research Funds for the Central Universities, China (Grant No. GK201803087, GK202003052, and GK202101003).

\section{Availability of data and materials}

The authors declare that the data supporting the finding of this study are available in the article and its supplementary information files. The raw sequencing reads data were deposited to NCBI as part of the BioProject PRJNA314730 via Sequence Read Archive (SRA). PacBio DNA-seq, Illumina DNA-seq, RNA-seq and Illumina DNA re-seq were available in SRR12874010, SRR12874011, SRR12874012, SRR12874013, SRR14041273, respectively.

\section{Declarations}

\section{Ethics approval and consent to participate}

Our team is a wildlife protection agency under the Shaanxi Academy of Sciences (China), cooperating and working with the authority department on Hongjian Nur for nearly 20 years, mainly devoted to the protection of the relict gull. Samples collection are performed in the daily conservation working following the institutional guidelines of the Nature Reserve Authority of Hongjian Nur. So, no extra permits are required for the collection of samples.

\section{Consent for publication}

Not applicable.

\section{Competing interests}

The authors declare that they have no competing interests.

Received: 21 October 2020 Accepted: 14 April 2021

Published online: 29 April 2021

\section{References}

1. Auezov EM. Taxonomic evaluation and systematic status of Larus relictus, Moscow. J Acad Sci 1971;50:235-242. (in Russian).

2. Yang C, Lian T, Wang Q, Huang Y, Xiao H. Preliminary study of genetic diversity and population structure of the relict Gull Larus relictus (Charadriiformes Laridae) using mitochondrial and nuclear genes. Mitochondrial DNA. 2016;27(6):4246-9. https://doi.org/10.3109/19401736.201 5.1022759 .

3. Liu Y, Lei JY, Zhang Y, Zhang ZW. The population, distribution and structure of relict Gull community in Bohai Bay, in: proceedings of the eighth National Congress of China ornithological society and the sixth ornithological symposium of the mainland and Taiwan in China. 2005.

4. Yin L, Fei JL, Liu CY. Birds of Hong Kong and South China. 8th ed. Hong Kong: Hong Kong Printing Department; 1994.

5. Liu D, Zhang G, Jiang H, Chen L, Meng D, Lu J. Seasonal dispersal and longitudinal migration in the relict Gull Larus relictus across the innerMongolian plateau. Peer J. 2017;5:e3380. https://doi.org/10.7717/peerj.3380.

6. Ikhumhen HO, Li TX, Lu SL. Assessment of a novel data driven habitat suitability ranking approach for Larus relictus specie using remote sensing and GIS. Ecol Model. 2020;432:109-221.

7. Zhang L, Li S, Luo J, Du P, Wu L, Li Y, et al. Chromosome-level genome assembly of the predator Propylea japonica to understand its tolerance to insecticides and high temperatures. Mol Ecol Resour. 2020;20(1):292-307. https://doi.org/10.1111/1755-0998.13100.

8. Yang C, Lian T, Wang Q, Huang Y, Xiao H. Structural characteristics of the relict Gull (Larus relictus) mitochondrial DNA control region and its comparison to other Laridae. Mitochond DNA A DNA. 2016;27(4):2487-91. https://doi.org/10.3109/19401736.2015.1033711.

9. Yang C, Wang Q, Huang Y, Xiao H. Complete mitochondrial genome of relict Gull, Larus relictus (Charadriiformes: Laridae). Mitochondrial DNA. 2016; 27(1):411-2. https://doi.org/10.3109/19401736.2014.898282

10. Kwon YS, Kim JH, Choe JC, Park YC. Low resolution of mitochondrial COI barcodes for identifying species of the genus Larus (Charadriiformes: Laridae). Mitochondrial DNA. 2012;23(2):157-66. https://doi.org/10.3109/194 01736.2012 .660921$.

11. Bian L, Li F, Ge J, Wang P, Chang Q, Zhang S, et al. Chromosome-leve genome assembly of the greenfin horse-faced filefish (Thamnaconus septentrionalis) using Oxford Nanopore PromethION sequencing and hi-C technology. Mol Ecol Resour. 2020;20(4):1069-79. https://doi.org/10.1111/1 755-0998.13183.

12. Giordano F, Aigrain L, Quail MA, Coupland P, Bonfield J, Davies R, et al. De novo yeast genome assemblies from MinION, PacBio and MiSeq platforms. Sci Rep. 2017;7(1):3935. https://doi.org/10.1038/s41598-017-03996-z.

13. Rhoads A, Au KF. PacBio sequencing and its applications. Genom Proteom Bioinf. 2015;13(5):278-89. https://doi.org/10.1016/j.gpb.2015.08.002.

14. Warren W, Hillier L, Tomlinson C, Minx P, Kremitzki M, Graves T, et al. A new chicken genome assembly provides insight into avian genome structure. G3 (Bethesda). 2017;7(1):109-17.

15. Peñalba JV, Deng Y, Fang Q, Joseph L, Moritz C, Cockburn A. Genome of an iconic Australian bird: high-quality assembly and linkage map of the superb fairy-wren (Malurus cyaneus). Mol Ecol Resour. 2020;20(2):560-78. https://doi. org/10.1111/1755-0998.13124.

16. Zhang G, Li C, Li Q, Li B, Larkin DM, Lee C, et al. Comparative genomics reveals insights into avian genome evolution and adaptation. Science. 2014; 346(6215):1311-20. https://doi.org/10.1126/science.1251385.

17. Feng SH, Stiller J, Deng Y, Armstrong J, Fang Q, Reeve AH, et al. Dense sampling of bird diversity increases power of comparative genomics. Nature. 2020;587(7833):252-7. https://doi.org/10.1038/s41586-020-2873-9.

18. Dimmer EC, Huntley RP, Alam-Faruque Y, Sawford T, O'Donovan C, Martin MJ, Bely B, Browne P, Chan WM, Eberhardt R. The UniProt-GO annotation database in 2011. Nucleic Acids Res, 2012;40(Database issue):D565-D570, DOl: https://doi.org/10.1093/nar/gkr1048.

19. Kanehisa M, Goto S. KEGG: Kyoto encyclopedia of genes and genomes. Nucleic Acids Res. 2000;28(1):27-30. https://doi.org/10.1093/nar/28.1.27. 
20. Tatusov RL, Natale DA, Garkavtsev IV, Tatusova TA, Shankavaram UT, Rao BS, et al. The COG database: new developments in phylogenetic classification of proteins from complete genomes. Nucleic Acids Res. 2001;29(1):22-8. https://doi.org/10.1093/nar/29.1.22.

21. Boeckmann B, Bairoch A, Apweiler R, Blatter MC, Estreicher A, Gasteiger E, et al. The SWISS-PROT protein knowledgebase and its supplement TrEMBL in 2003. Nucleic Acids Res. 2003;31(1):365-70. https://doi.org/10.1093/nar/ gkg095.

22. Marchler-Bauer A, Lu S, Anderson JB, Chitsaz F, Derbyshire MK, DeWeeseScott C, Fong JH, Geer LY, Geer RC, Gonzales NR. CDD: a conserved domain database for the functional annotation of proteins. Nucleic Acids Res, 2011; 39(Database issue):D225-D229, DOl: https://doi.org/10.1093/nar/gkq1189.

23. Gentles AJ, Wakefield MJ, Kohany O, Gu WJ, Batzer MA, Pollock DD, et al. Evolutionary dynamics of transposable elements in the short-tailed opossum Monodelphis domestica. Genome Res. 2007;17(7):992-1004. https://doi.org/10.1101/gr.6070707.

24. Bohne A, Brunet F, Galiana-Arnoux D, Schultheis C, Volff JN. Transposable elements as drivers of genomic and biological diversity in vertebrates. Chromosom Res. 2008;16(1):203-15. https://doi.org/10.1007/s10577-007-12 02-6.

25. Yang C, Wang QX, Huang Y, Xiao H. Phylogenetic relationships of gulls based on the complete sequence of mitochondrial Cytb gene. Acta Zootaxon Sin. 2013;38(2):225-38.

26. Pons JM, Hassanin A, Crochet PA. Phylogenetic relationships within the Laridae (Charadriiformes: Aves) inferred from mitochondrial markers. Mol Phylogenet Evol. 2005;37(3):686-99. https://doi.org/10.1016/j.ympev.2005.05. 011.

27. Baker AJ, Pereira SL, Paton TA. Phylogenetic relationships and divergence times of Charadriiformes genera: multigene evidence for the cretaceous origin of at least 14 clades of shorebirds. Biol Lett. 2007;3(2):205-9. https:// doi.org/10.1098/rsbl.2006.0606.

28. Hung CM, Shaner PJL, Zink RM, Liu WC, Chu TC, Huang WS, et al. Drastic population fluctuations explain the rapid extinction of the passenger pigeon. Proc Natl Acad Sci USA. 2014;111(29):10636-41. https://doi.org/10.1 073/pnas.1401526111.

29. Grabherr MG, Haas BJ, Yassour M, Levin JZ, Thompson DA, Amit I, et al. Fulllength transcriptome assembly from RNA-Seq data without a reference genome. Nat Biotechnol. 2011;29(7):644-52. https://doi.org/10.1038/nbt.1 883.

30. Salmela L, Rivals E. LoRDEC: accurate and efficient long read error correction. Bioinformatics. 2014;30(24):3506-14. https://doi.org/10.1093/ bioinformatics/btu538.

31. Kajitani R, Toshimoto K, Noguchi H, Toyoda A, Ogura Y, Okuno M, et al. Efficient de novo assembly of highly heterozygous genomes from wholegenome shotgun short reads. Genome Res. 2014;24(8):1384-95. https://doi. org/10.1101/gr.170720.113.

32. Ye C, Hill C, Wu S, Ruan J, Ma Z. DBG2OLC: efficient assembly of large genomes using long erroneous reads of the third generation sequencing technologies. Sci Rep. 2016:6(1):31900. https://doi.org/10.1038/srep31900.

33. Chin CS, Peluso P, Sedlazeck FJ, Nattestad M, Concepcion GT, Clum A, et al. Phased diploid genome assembly with single-molecule real-time sequencing. Nat Methods. 2016;13(12):1050-4. https://doi.org/10.1038/nmeth.4035.

34. Parra G, Bradnam K, Korf I. CEGMA: a pipeline to accurately annotate core genes in eukaryotic genomes. Bioinformatics. 2007;23(9):1061-7. https://doi. org/10.1093/bioinformatics/btm071.

35. Haas BJ, Salzberg SL, Zhu W, Pertea M, Allen JE, Orvis J, et al. Automated eukaryotic gene structure annotation using evidence modeler and the program to assemble spliced alignments. Genome Biol. 2008;9(1):R7. https:// doi.org/10.1186/gb-2008-9-1-r7.

36. Burge C, Karlin S. Prediction of complete gene structures in human genomic DNA. J Mol Biol. 1997;268(1):78-94. https://doi.org/10.1006/jmbi.1 997.0951.

37. Stanke M, Waack S. Gene prediction with a hidden Markov model and a new intron submodel. Bioinformatics. 2003;19(Suppl 2):215-25.

38. Majoros WH, Pertea M, Salzberg SL. TigrScan and GlimmerHMM: two open source ab initio eukaryotic gene-finders. Bioinformatics. 2004;20(16):2878-9. https://doi.org/10.1093/bioinformatics/bth315.

39. Blanco E, Parra G, Guigó R. Using geneid to identify genes. Curr Protoc Bioinform. 2007:4(4.3):1-28.

40. Korf I. Gene finding in novel genomes. BMC Bioinform. 2004;5(1):59. https:// doi.org/10.1186/1471-2105-5-59.
41. Keilwagen J, Wenk M, Erickson JL, Schattat MH, Jan G, Frank H. Using intron position conservation for homology-based gene prediction. Nucleic Acids Res. 2016;44(9):e89. https://doi.org/10.1093/nar/gkw092.

42. Pertea M, Kim D, Pertea GM. Transcript-level expression analysis of RNA-seq experiments with HISAT, StringTie and Ballgown. Nat Protoc. 2016;11(9): 1650-67. https://doi.org/10.1038/nprot.2016.095.

43. Haas BJ, Papanicolaou A. TransDecoder (find coding regions within transcripts). Github, nd https://github.com/TransDecoder/TransDecoder. 2015. v 5.0.1, released on 13 Sep 2017.

44. Tang S, Lomsadze A, Borodovsky M. Identification of protein coding regions in RNA transcripts. Nucleic Acids Res. 2015;43(12):e78. https://doi.org/10.1 093/nar/gkv227.

45. Campbell MA, Haas BJ, Hamilton JP, Mount SM, Buell CR. Comprehensive analysis of alternative splicing in rice and comparative analyses with Arabidopsis. BMC Genomics. 2006;7(1):327. https://doi.org/10.1186/1471-2164-7-327.

46. Xu Z, Wang H. LTR_FINDER: an efficient tool for the prediction of full-length LTR retrotransposons. Nucleic Acids Res. 2007;35(suppl. 2):W265-8. https:// doi.org/10.1093/nar/gkm286.

47. Han Y, Wessler SR. MITE-hunter: a program for discovering miniature inverted-repeat transposable elements from genomic sequences. Nucleic Acids Res. 2010;38(22):e199. https://doi.org/10.1093/nar/gkq862.

48. Price $A L$, Jones NC, Pevzner PA. De novo identification of repeat families in large genomes. Bioinformatics. 2005;21(Suppl 1):i351-8. https://doi.org/10.1 093/bioinformatics/bti1018.

49. Edgar RC, Myers EW. PILER: identification and classification of genomic repeats. Bioinformatics. 2005;21(Suppl 1):i152-8. https://doi.org/10.1093/ bioinformatics/bti1003.

50. Jurka J, Kapitonov W, Pavlicek A, Klonowski P, Kohany O, Walichiewicz J. Repbase update, a database of eukaryotic repetitive elements. Cytogenet Genome Res. 2005;110(1-4):462-7. https://doi.org/10.1159/000084979.

51. Tarailo-Graovac M, Chen N. Using RepeatMasker to identify repetitive elements in genomic sequences. Curr Protoc Bioinform, 2009; Suppl 5 (4. 10):1-14.

52. Griffiths-Jones S, Moxon S, Marshall M, Khanna A, Eddy SR, Bateman A. Rfam: annotating non-coding RNAs in complete genomes. Nucleic Acids Res. 2005:33(Database issue):D121-4. https://doi.org/10.1093/nar/gki081.

53. Griffiths-Jones S, Grocock RJ, van Dongen S, Bateman A, Enright AJ. miRBase: microRNA sequences, targets and gene nomenclature. Nucleic Acids Res, 2006;34(Database issue):D140-D144, DOl: https://doi.org/10.1093/ nar/gkj112.

54. Nawrocki EP, Eddy SR. Infernal 1.1: 100-fold faster RNA homology searches. Bioinformatics. 2013;29(22):2933-5. https:/doi.org/10.1093/bioinformatics/ btt509.

55. Lowe TM, Eddy SR. tRNAscan-SE: a program for improved detection of transfer RNA genes in genomic sequence. Nucleic Acids Res. 1997;25(5): 955-64. https://doi.org/10.1093/nar/25.5.955.

56. She R, Chu JSC, Wang K, Pei J, Chen NS. GenBlastA: enabling BLAST to identify homologous gene sequences. Genome Res. 2009;19(1):143-9. https://doi.org/10.1101/gr.082081.108.

57. Birney E, Clamp M, Durbin R. GeneWise and genomewise. Genome Res. 2004;14(5):988-95. https://doi.org/10.1101/gr.1865504.

58. Altschul SF, Gish W, Miller W, Myers EW, Lipman DJ. Basic local alignment searchtool. J Mol Biol. 1990;215(3):403-10. https://doi.org/10.1016/S0022-283 6(05)80360-2.

59. Emms DM, Kelly S. OrthoFinder: phylogenetic orthology inference for comparative genomics. Genome Biol. 2019;20(1):1-14.

60. Yu G, Wang LG, Han Y, He QY. clusterProfiler: an R package for comparing biological themes among gene clusters. Omics. 2012;16(5):284-7. https:// doi.org/10.1089/omi.2011.0118.

61. Katoh K, Asimenos G, Toh H. Multiple alignment of DNA sequences with MAFFT. Bioinform DNA Seq Anal Hum Press. 2009:39-64. https://doi.org/1 0.1007/978-1-59745-251-9_3.

62. Suyama M, Torrents D, Bork P. PAL2NAL: robust conversion of protein sequence alignments into the corresponding codon alignments. Nucleic Acids Res. 2006;34(suppl 2):609-12.

63. Talavera G, Castresana J. Improvement of phylogenies after removing divergent and ambiguously aligned blocks from protein sequence alignments. Syst Biol. 2007:56(4):564-77. https://doi.org/10.1080/10635150701472164.

64. Kalyaanamoorthy S, Minh BQ, Wong TK, von Haeseler A, Jermiin LS. ModelFinder: fast model selection for accurate phylogenetic estimates. Nat Methods. 2017;14(6):587-9. https://doi.org/10.1038/nmeth.4285. 
65. Nguyen LT, Schmidt HA, Von Haeseler A, Minh BQ. IQ-TREE: a fast and effective stochastic algorithm for estimating maximum-likelihood phylogenies. Mol Biol Evol. 2015;32(1):268-74. https://doi.org/10.1093/ molbev/msu300.

66. Yang Z. PAML: a program package for phylogenetic analysis by maximum likelihood. Bioinformatics. 1997;13(5):555-6. https://doi.org/10.1093/ bioinformatics/13.5.555.

67. Li H, Durbin R. Inference of human population history from individual whole-genome sequences. Nature. 2011;475(7357):493-6. https://doi.org/1 $0.1038 /$ nature10231.

\section{Publisher's Note}

Springer Nature remains neutral with regard to jurisdictional claims in published maps and institutional affiliations.

Ready to submit your research? Choose BMC and benefit from:

- fast, convenient online submission

- thorough peer review by experienced researchers in your field

- rapid publication on acceptance

- support for research data, including large and complex data types

- gold Open Access which fosters wider collaboration and increased citations

- maximum visibility for your research: over $100 \mathrm{M}$ website views per year

At BMC, research is always in progress.

Learn more biomedcentral.com/submissions 